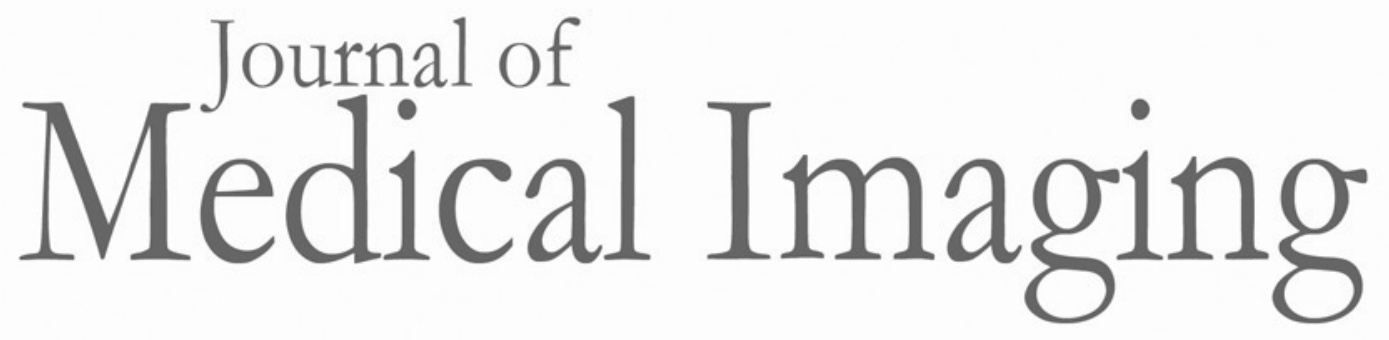

\title{
Three-dimensionally-printed anthropomorphic physical phantom for mammography and digital breast tomosynthesis with custom materials, lesions, and uniform quality control region
}

\author{
Andrea H. Rossman \\ Matthew Catenacci \\ Christine Zhao \\ Dhiraj Sikaria \\ John E. Knudsen \\ Danielle Dawes \\ Michael E. Gehm \\ Ehsan Samei \\ Benjamin J. Wiley \\ Joseph Y. Lo
}




\title{
Three-dimensionally-printed anthropomorphic physical phantom for mammography and digital breast tomosynthesis with custom materials, lesions, and uniform quality control region
}

\author{
Andrea H. Rossman, ${ }^{a, b}$ Matthew Catenacci, ${ }^{c}$ Christine Zhao, ${ }^{a, b, d}$ Dhiraj Sikaria, ${ }^{a, b}$ John E. Knudsen, ${ }^{a, b}$ \\ Danielle Dawes, ${ }^{a, b}$ Michael E. Gehm, ${ }^{d}$ Ehsan Samei, ${ }^{a, b, d}$ Benjamin J. Wiley, ${ }^{c}$ and Joseph Y. Lo ${ }^{a, b, d, *}$ \\ ${ }^{a}$ Duke University, Department of Biomedical Engineering, Durham, North Carolina, United States \\ ${ }^{\mathrm{b}}$ Duke University School of Medicine, Carl E. Ravin Advanced Imaging Laboratories, Department of Radiology, Durham, North Carolina, \\ United States \\ 'Duke University, Department of Chemistry, Durham, North Carolina, United States \\ dDuke University, Department of Electrical and Computer Engineering, Durham, North Carolina, United States
}

\begin{abstract}
Anthropomorphic breast phantoms mimic patient anatomy in order to evaluate clinical mammography and digital breast tomosynthesis system performance. Our goal is to create a modular phantom with an anthropomorphic region to allow for improved lesion and calcification detection as well as a uniform region to evaluate standard quality control (QC) metrics. Previous versions of this phantom used commercial photopolymer inkjet three-dimensional printers to recreate breast anatomy using four surfaces that were fabricated with commercial materials spanning only a limited breast density range of $36 \%$ to $64 \%$. We use modified printers to create voxelized, dithered breast phantoms with continuous gradations between glandular and adipose tissues. Moreover, the new phantom replicates the low-end density (representing adipose tissue) using third party material, Jf Flexible, and increases the high-end density to the density of glandular tissue and beyond by either doping Jf Flexible with salts and nanoparticles or using a new commercial resin, VeroPureWhite. An insert design is utilized to add masses, calcifications, and iodinated objects into the phantom for increased utility. The uniform chest wall region provides a space for traditional QC objects such as line pair patterns for measuring resolution and scale bars for measuring printer linearity. Incorporating these distinct design modules enables us to create an improved, complete breast phantom to better evaluate clinical mammography systems for lesion and calcification detection and standard QC performance evaluation. ( 2019 Society of Photo-Optical Instrumentation Engineers (SPIE) [DOI: 10.1117/1.JMI.6.2.021604]
\end{abstract}

Keywords: imaging phantom; mammography; digital breast tomosynthesis; three-dimensional printing; breast cancer.

Paper 18192SSR received Sep. 2, 2018; accepted for publication Mar. 1, 2019; published online Mar. 22, 2019.

\section{Introduction}

\subsection{Background}

Physical phantoms allow for direct evaluation of different imaging systems and settings. Full-field digital mammography (FFDM) phantoms currently in clinical use, such as the American College of Radiology (ACR) ${ }^{1}$ and Contrast Detail Mammography (CDMAM) ${ }^{2}$ phantoms, are composed of simple test objects embedded in a single layer within a uniform background. Although widely accepted, these simple phantoms may not directly predict how the hardware-software combination of a medical imaging system will image a variety of patients in the clinic. In recent years, digital breast tomosynthesis (DBT) adoption has been accelerating, with DBT installations comprising half of all US mammography facilities and accounting for a third of all units. ${ }^{3}$ However, existing clinical breast phantoms all predate DBT and do not evaluate how image quality can be impacted by DBT artifacts and three-dimensional (3-D) anatomical details. ${ }^{4}$

To address the limitations of existing clinical phantoms, anthropomorphic phantoms mimic real anatomy to evaluate the quality of the image. For example, breast texture is represented by a complex, marbled background in the CIRS BR3D Model 020 (Computerized Imaging Reference Systems, Inc., Norfolk, Virginia, hereafter CIRS). The Penn phantom uses a combination of 3-D printing and manual backfilling to create a more realistic 3-D texture, ${ }^{5}$ and other phantoms have also been proposed with different textures. ${ }^{6-10}$ However, these heterogeneous phantoms are generated randomly or procedurally, and therefore, may not accurately or reproducibly represent real anatomy. Since the postprocessing and/or reconstruction algorithms of imaging devices are optimized for actual anatomy, phantoms should reproduce that anatomy as faithfully as possible. Threedimensional printing is an emerging phantom fabrication method for achieving realistic anatomy and texture but has not been used for mammography phantoms as frequently as it has been used for other imaging modalities. ${ }^{11}$

\subsection{Previous Work}

To address the aforementioned gap, physical phantoms can also be based on actual anatomy from medical imaging. This study is the first to create physical phantoms using the XCAT Breast Phantom Cohort II (Duke University, Durham, NC), in which 
patient breast CT images were transformed into voxelized, computational models. ${ }^{12-14}$ We previously made multimaterial 3-D printed breast phantoms that were first binary ${ }^{15}$ and then four mesh surfaces for improved resolution. ${ }^{16}$ There were no lesions included in the previous work with the exception of two-dimensional (2-D) inserts. ${ }^{17}$ Voxelization was used to create a liver CT phantom, but this technique was not applied to breast phantoms. ${ }^{18}$ The goal of this study was to create an anthropomorphic breast phantom that accurately represented breast texture, attenuation, and shape. An additional aim was to provide modular options including realistic lesions in interchangeable inserts and a uniform space to evaluate standard quality control (QC) metrics.

\section{Methods}

\subsection{Printer Technology}

For creating medical imaging phantoms, photopolymer inkjet printers are attractive because of their high resolution and the potential for material customization via ink doping. Following conventions for 3-D printing, previous studies required converting voxelized imaging data to mesh surfaces, then relying upon the printing software to convert those surfaces back into voxelized printing instructions. ${ }^{15,16}$ However, translating high-resolution breast phantom data into surfaces required an impractical amount of computer memory and resulted in the loss of small details and boundary transitions. We, therefore, bypassed these intermediate steps to allow direct, voxelized printing for two printers: the Objet Connex 350 and the Objet Eden 333 (Stratasys Ltd., Rehovot, Israel, hereafter Stratasys). ${ }^{18,19}$ Custom software was written in MATLAB (MathWorks Inc., Natick, MA) to reverse engineer the communications protocol used to control each printer, thus allowing a direct, voxel-to-voxel mapping from virtual to physical domains. Furthermore, digital dithering allowed arbitrary mixes of the materials to provide smooth transitions and intermediate densities. In this manner, each source volume data were dithered and 3-D interpolated to the nominal voxel pitch of each printer, which was $42 \times 84 \mu \mathrm{m}$ in plane but with different slice thicknesses of $16 \mu \mathrm{m}$ for the Eden and $30 \mu \mathrm{m}$ for the Connex. The Connex can print with two materials and support at the same time. The Eden was designed to print with only one material and support; therefore, in order to print with two materials, the Eden support cartridge was replaced with the desired second material. The Eden was further modified to facilitate testing of third-party and custom-made materials.

\subsection{Material Investigation}

In order to accurately mimic the radiographic density of breast tissue, photopolymer inkjet materials were investigated. In previous versions of this phantom, the commercial materials TangoPlus and VeroWhitePlus (Stratasys) were, respectively, used to represent adipose and fibroglandular tissue. ${ }^{15,16}$ Based on the lower cost and simpler formulations of third party inks, Jf Flexible resin (Molecule Digital LLC, Concord, California) was investigated as an alternative for adipose tissue. To mimic the attenuation of fibroglandular tissue, Jf Flexible resin was also doped with 3 weight percent (wt. \%) zinc acetate or $1.6 \mathrm{wt}$. \% tungsten nanopowder (US Research Nanomaterials Inc., Houston, Texas). The new commercial material VeroPureWhite (Stratasys) was also used to represent glandular tissue, not to be confused with the older VeroWhitePlus. Attenuation and scatter were both measured with a Selenia Dimensions system (Hologic Inc., Marlborough, Massachusetts) in conventional mammography mode using a target/filter of $\mathrm{W} / \mathrm{Rh}$ at $28 \mathrm{kVp}$ with the grid. As in a previous study, ${ }^{15}$ the linear attenuation coefficients $(\mu)$ were determined using additional beam filtration at the tube exit port by $4 \mathrm{~cm}$ of Model 014 50/50 tissue equivalent slabs (CIRS) to achieve a typical mammography spectrum. The linearity of the attenuation based on dopant concentration was determined using the digital dithering technique to combine doped versus pure Jf Flexible to create different weight percentages. Scatter fractions were measured for $4-\mathrm{cm}$ thick slabs using beam stops of decreasing sizes and corrected for effects of finite beam stop size, field nonuniformity, and detector cover scatter. 3-D-printed slabs of TangoPlus and VeroPureWhite were selected to represent photopolymers spanning the range of densities and were compared to Model 014 reference adipose and glandular blocks (CIRS).

\subsection{Phantom Fabrication}

The physical phantom was created from a virtual XCAT Breast Phantom Cohort II case (Duke University, Durham, North Carolina). Using human subjects with normal breast anatomy, dedicated breast CT scans were performed with a Koning Breast CT system (Koning Corporation, West Henrietta, New York). Each 3 -D volume of $155 \times 155 \times 155 \mu$ m voxels was segmented at the same resolution into a virtual phantom with five classes to represent $0 \%$ to $100 \%$ volumetric breast density, defined as the percentage of fibroglandular tissue by volume (with skin mapped to $100 \%)^{12-14}$ and was compressed with a finite-element method to simulate mammographic compression. ${ }^{15}$ The phantom for this study reproduced the thickness and shape of a 4-cm compressed breast, i.e., the phantom material was the equivalent thickness as real tissue. Following initial evaluations of attenuation, TangoPlus or Jf Flexible was used for adipose tissue, whereas VeroPureWhite or tungstendoped Jf Flexible was used for glandular tissue. These choices provided commercial and customized ink versions, respectively. The current phantom was designed as two butterflied halves but the design can be changed into a single breast volume for ease of handling or more thinner slabs to provide greater customization.

\subsection{Lesion Insert Design}

While our previous phantoms were limited to normal anatomy, the most recent design includes the option of masses, iodinated masses, and calcifications. Inserts of 0.5-cm thickness were 3-D printed with healthy anatomy with and without 64 masses in a contrast-detail pattern with Jf Flexible with and without tungsten. The contrast-detail pattern was composed of microlobulated masses chosen to represent a range of clinically relevant sizes and contrasts. ${ }^{20,21}$ The masses were arranged in four arrays of $4 \times 4$ microlobulated masses spanning in one direction $0.2-$, $0.3-, 0.4-$, and $0.5-\mathrm{mm}$ diameter, and in the other direction $100 \%, 104 \%, 107 \%$, and $110 \%$ attenuation relative to the fibroglandular tissue in that phantom. For contrast-enhanced mammography, the same contrast-detail pattern was printed in a uniform background of Jf Flexible, with lesions represented by $2,4,6$, and 8 wt. \% iodobutane (equivalent to $15,30,45$, and $60 \mathrm{mg} / \mathrm{mL}$ of iodine) to demonstrate the capability to deliver high iodine concentrations. A simpler design was composed of the slab of normal anatomy but with cylindrical holes that 
could hold existing lesion targets, such as cylinders with different levels of iodine provided by CIRS. The Hologic Selenia Dimensions system used did not have dual energy capability, so the phantom was imaged using $\mathrm{W} / \mathrm{Rh}$ at $28 \mathrm{kVp}$ in mammography mode.

The calcification insert was made by crushing eggshells in a mortar and pestle, sifting with a fine mesh strainer, and finally fixing the particles between two pieces of adhesive tape with dimensions $4.2 \mathrm{~cm} \times 4.8 \mathrm{~cm}$. Calcifications were evaluated using a commercial flatbed scanner at 2400 dpi (Epson America Inc., Long Beach, California). This was treated as a 2-D insert and imaged in a homogenous, tissue-equivalent background for evaluation.

\subsection{Chest Wall with QC Metrics}

The uniform chest wall region was designed to be customizable to include conventional QC test objects typically found in conventional, uniform phantoms. Prior to printing, a $35-\mathrm{mm}$ piece of uniform "adipose" region was added to the chest wall of each breast slab, as shown in Fig. 1. Several hollow (air) features were created, including 3.35-mm diameter hollow columns for alignment and assembly of the slabs, and $2.5-\mathrm{mm}$ hollow columns and $1.0-\mathrm{mm}$ hollow spheres to act as air contrast fiducial markers. Solid, embedded features included a "glandular" sphere and rectangular prism for uniformity, geometric distortion, and signal-to-noise ratio, and a scale bar ranging from adipose to glandular in $20 \%$ increments to test the linearity of contrast.

In addition to 3-D-printed features, metal ink stickers were created as 2-D inserts using a commercial printing service (MorningPrint Inc., Irvine, California). The stickers were designed with line pair starburst patterns, circle and square test objects to evaluate modulation transfer function (MTF) or artifact spread function (ASF), and fiducial markers. The line pairs range from 1.5 to $10 \mathrm{lp} / \mathrm{mm}$ and are intended for technologists to make quick, visual assessments. They also demonstrate the flexibility, robustness, and high resolution of the metal ink printing process. These metrics are already well-established in other phantoms, therefore, they are only qualitatively demonstrated in the context of this hybrid design. As an example, the line pairs were used to evaluate and compare the resolution of the Hologic Selenia Dimensions system in four modes: contact mammography, $1.8 \times$ magnification view mammography, and

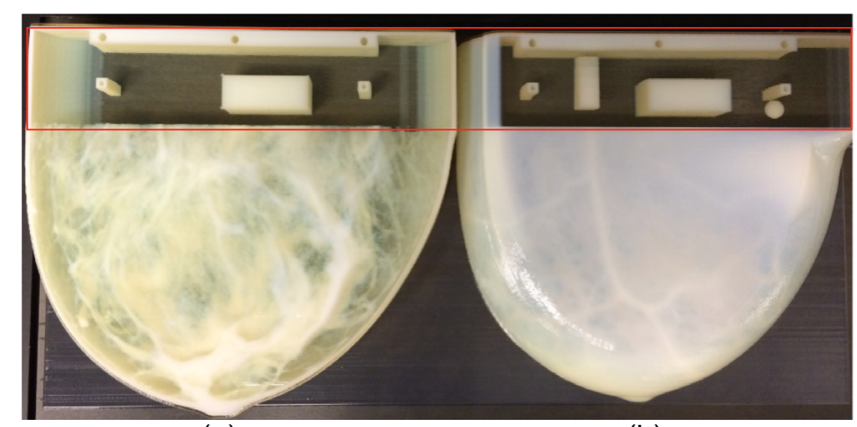

(a)

(b)

Fig. 1 Photograph of two halves of hybrid breast phantom: (a) inferior and (b) superior, with uniform chest wall region shown by red box that can contain conventional QC test objects to supplement the anthropomorphic anatomy section.
DBT parallel or perpendicular to tube motion direction. The resolution of each mode was determined based on the consensus of three observers. Fiducial markers were added to allow for potential automation of this process.

\section{Results}

All our phantoms use two materials to represent adipose versus glandular tissue, but there have been substantial improvements in the modeling of those tissue compartments, as shown in Fig. 2. In the first generation phantom, the volumetric data were binarized into adipose versus glandular surfaces, resulting in loss of small details and abrupt edges. ${ }^{15}$ The second generation phantom was segmented into four surfaces, which were printed using a commercially available option to create mixed, intermediate versions of the two extreme materials. ${ }^{16}$ Finally, the current study used the voxelized and dithered approach and also switched to the higher-resolution XCAT Cohort II data, providing the finest details and transitions between adipose and glandular regions.

Jf Flexible resin was doped with zinc acetate or tungsten to increase the attenuation. Figure 3 depicts the photopolymer resin doping results. With 3 wt. \% zinc acetate, printed samples achieved $\mu=0.68 / \mathrm{cm}$, matching the attenuation of fibroglandular tissue. In subsequent repeat prints, however, the attenuation dropped to $0.58 / \mathrm{cm}$, which suggested clogging or precipitation problems. Tungsten-doped Jf Flexible at $1.6 \mathrm{wt}$. \% achieved $\mu=0.75 / \mathrm{cm}$, which exceeded that of fibroglandular tissue. Although volumetric breast density is a fraction between $0 \%$ and $100 \%$, this material can be thought of as $140 \%$ on that scale. Since materials available to represent adipose tissue are limited to $\sim 40 \%$ volumetric breast density on the low end, this higher $140 \%$ density can qualitatively demonstrate the expected amount of visual contrast between adipose and glandular tissue. Another option to represent fibroglandular tissue is the new commercial material VeroPureWhite that was measured at $\mu=0.65 / \mathrm{cm}, 92 \%$ volumetric breast density.

Scatter fractions for $4 \mathrm{~cm}$ of printed material were $7.2 \%$ for TangoPlus and $7.4 \%$ for VeroPureWhite, compared to $6.0 \%$ for adipose and $6.2 \%$ for glandular reference materials. By substituting adipose with TangoPlus, or glandular with VeroPureWhite, the difference in scatter intensities would amount to only $1.2 \%$ of the background pixel values, compared

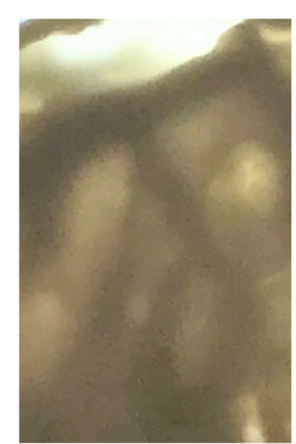

(a)

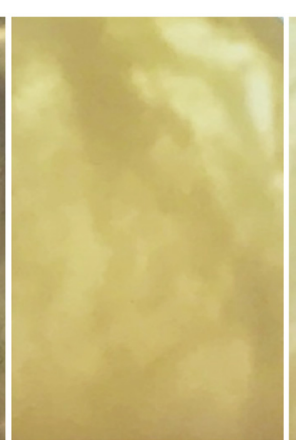

(b)

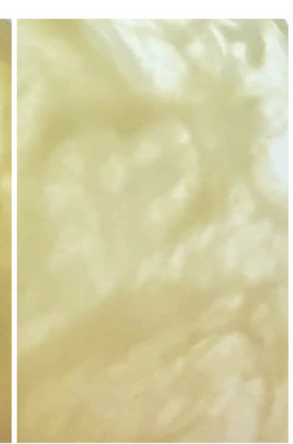

(c)
Fig. 2 Three generations of breast phantoms show increasing detail and smoother transitions: (a) binary surfaces, (b) four surfaces, and (c) voxelized and dithered. Images are close-up photographs of $25 \times 40 \mathrm{~mm}$, taken from a 10-mm-thick slab. 


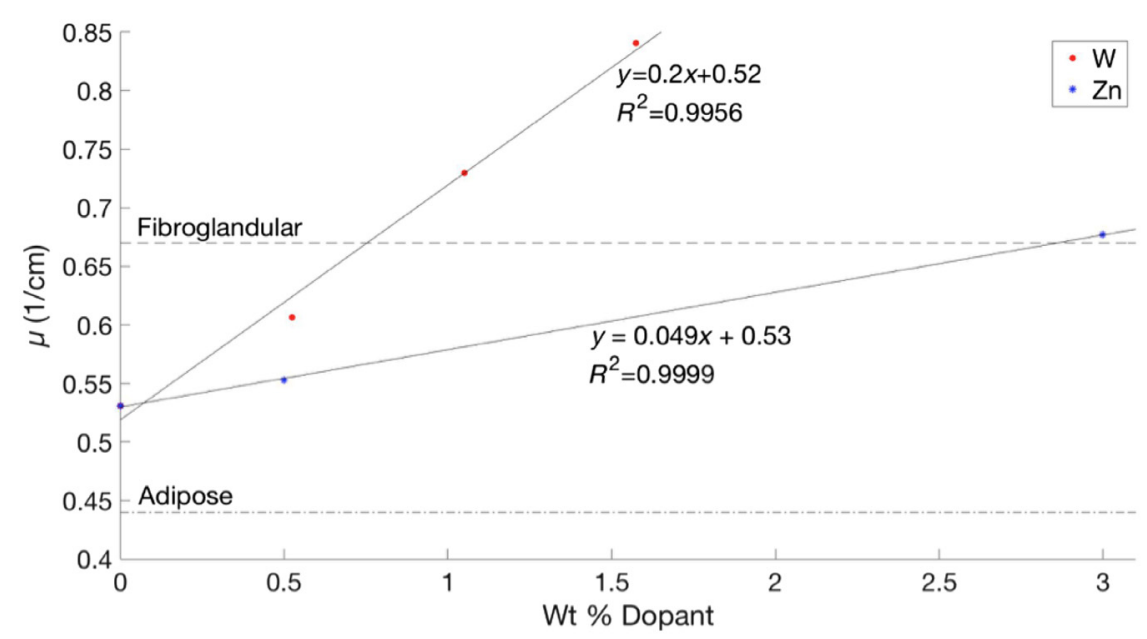

Fig. 3 Plot of linear attenuation coefficient versus dopant concentration for Jf Flexible resin doped with zinc acetate or tungsten, imaged at W/Rh $28 \mathrm{kVp}$ on the Hologic Selenia dimensions system. The horizontal dashed lines represent $0 \%$ and $100 \%$ fibroglandular tissue, $\mu=0.44$ and $\mu=0.67$, respectively.

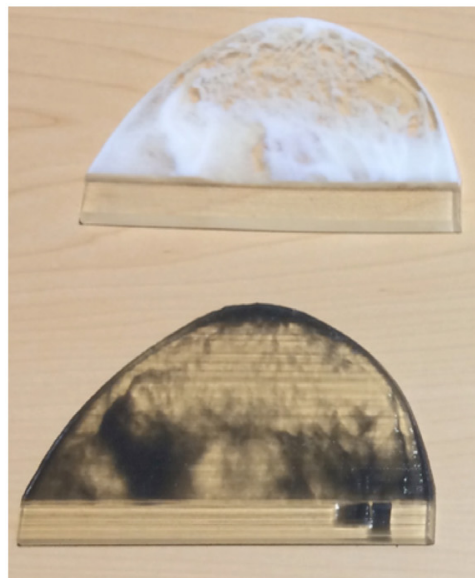

(a)

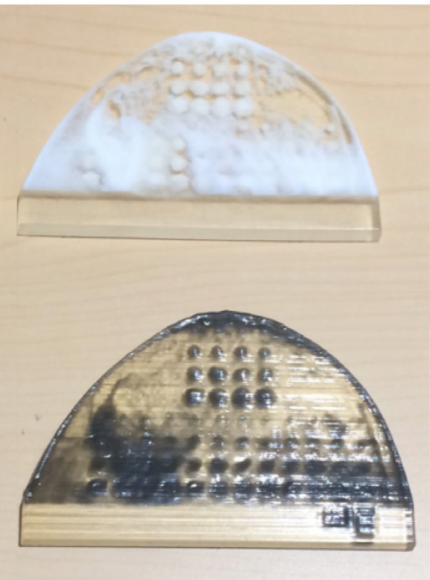

(b)

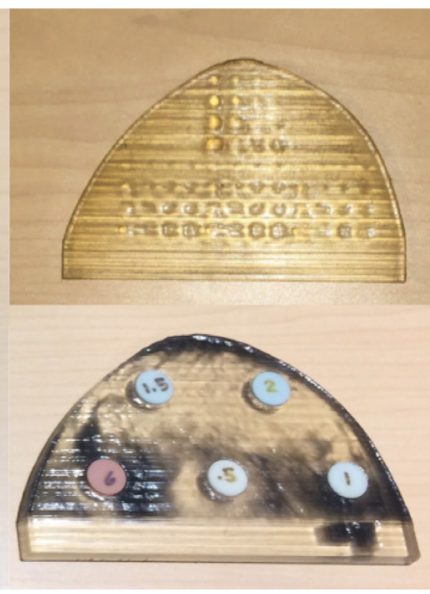

(c)

Fig. 4 Photographs of modular lesion insert design created with VeroPureWhite (white) or tungstendoped (black) materials: (a) healthy breast anatomy, (b) breast anatomy with masses in contrast-detail patterns without contrast, and (c) iodinated inserts with colorless iodobutane masses on top and CIRS iodinated disks on bottom.

to $1.8 \%$ for the background noise of an ACR accreditation phantom imaged on the same system under AEC conditions.

A modular insert design was developed as shown in Fig. 4, which enabled for the incorporation of lesions without and with iodinated contrast into both the tungsten and VeroPureWhite phantom versions.

Figure 5 shows both the tungsten and VeroPureWhite phantom versions portraying normal anatomy imaged on the Hologic system in mammography and DBT modes. Note the higher visual contrast offered by the tungsten version.

Figure 6 displays the same phantoms but with the center $0.5-\mathrm{cm}$ slab of normal anatomy replaced the lesion insert with addition of masses in four sets of contrast-detail patterns. Within each $4 \times 4$ set of masses, each of the 16 masses has unique morphology that can be adjusted parametrically and vary in contrast parallel to the chest wall and size in the chest wall to nipple direction (left to right as shown in Fig. 6).

Figure 7 demonstrates the imaging of the phantom with lesion inserts featuring targets with iodine. As before, the mass contrast-detail pattern clearly shows the range of contrasts and sizes of the masses, and the CIRS iodinated cylinders also show differing levels of conspicuity. Since this clinical system was not optimized for contrast-enhanced imaging, these images were taken at the maximum energy allowed for mammography and DBT to demonstrate iodine signal qualitatively.

The calcification insert images in a uniform, tissue-equivalent background show the expected decrease in calcification detection with mammography and DBT imaging compared to the optical scan ground truth (Fig. 8). Based on that optical scan, the insert contained 276 measurable calcifications with major axis length ranging from 53 to $710 \mu \mathrm{m}$ with an average size of $200 \mu \mathrm{m}$. These sizes are clinically relevant to typical breast calcifications that range from 50 to $500 \mu \mathrm{m} .^{22}$ Based on this subjective experience, a breast imaging radiologist confirmed that the radiographic appearance realistically mimicked pleomorphic or coarse heterogeneous morphology.

When the line pair stickers were also imaged in a uniform, tissue-equivalent background, there was a similar, expected 


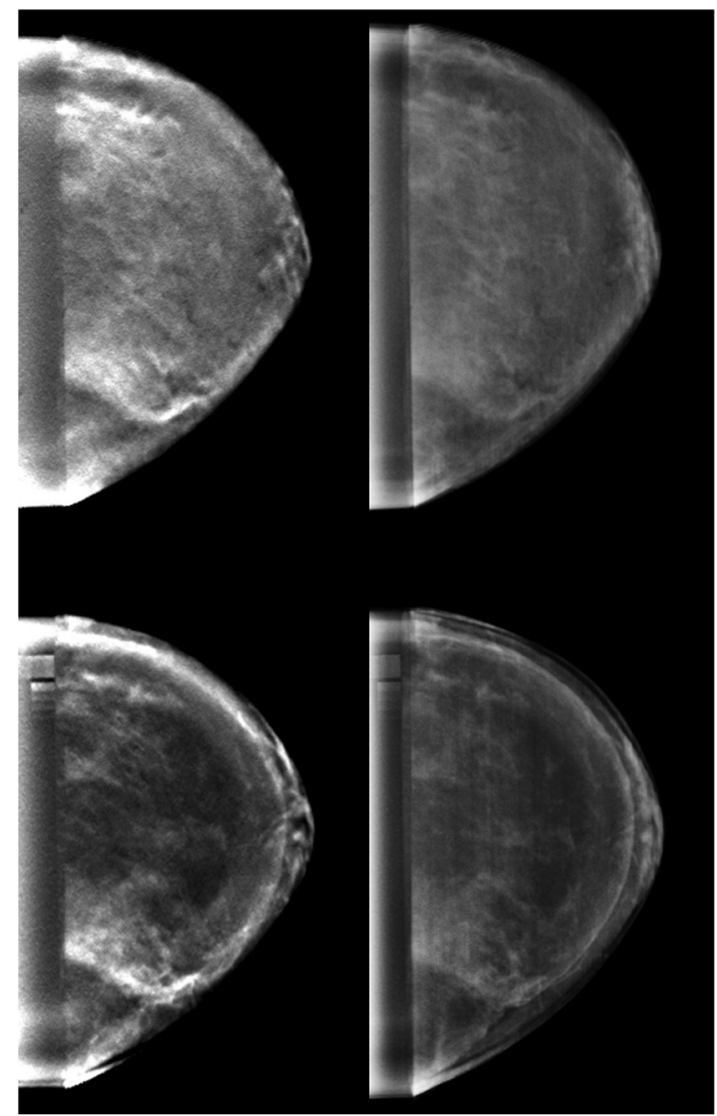

(a)

(b)

Fig. 5 Breast phantoms with normal anatomy printed with (a) and (b) Jf Flexible to mimic adipose tissue and VeroPureWhite and (c) and (d) tungsten-doped Jf Flexible to mimic fibroglandular tissue. (a) and (c) A DBT reconstructed slice image of both breasts and (b) and (d) mammography image of both breasts.

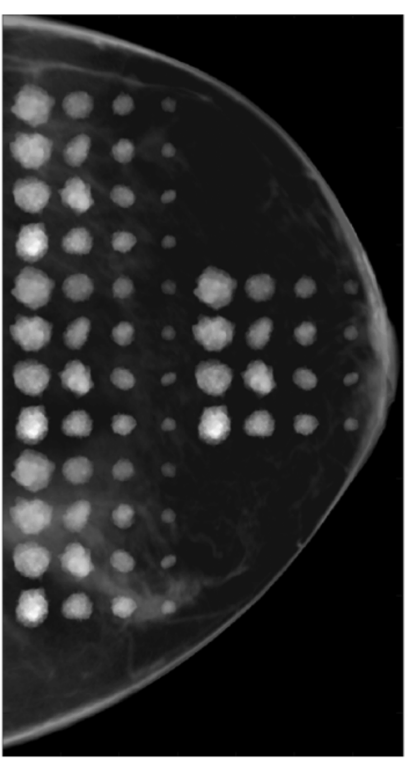

(a)

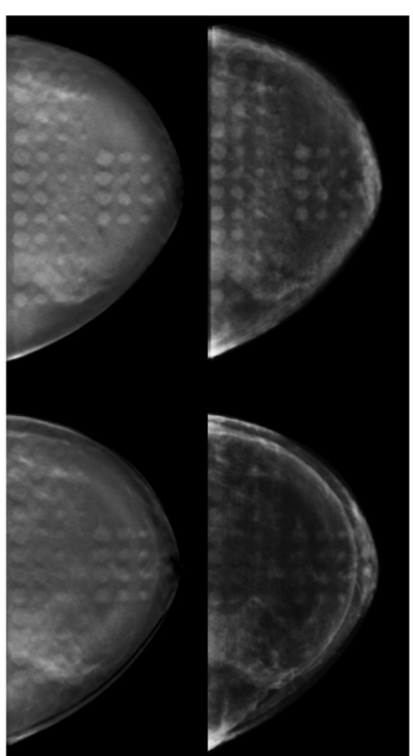

(b) (c)
Fig. 6 Breasts imaged with the mass contrast-detail insert. Tungstendoped breast on the top and VeroPureWhite breast on the bottom. (a) Simulated mammography image of the virtual phantom containing the lesion insert with exaggerated contrast to illustrate lesion location and details, (b) DBT reconstructed slice image of the phantoms, and (c) mammography image of the phantoms. decrease in resolution from mammography and magnification view at $6.5 \mathrm{lp} / \mathrm{mm}$ to DBT images at $4 \mathrm{lp} / \mathrm{mm}$ when oriented either parallel or orthogonal to the tube motion direction (Fig. 9). The larger objects were designed to evaluate quantitative metrics such as MTF and ASF, but that is beyond the scope of this work.

\section{Discussion}

For decades, assessment of mammographic image quality relied upon subjective scoring of intentionally simplistic, uniform phantoms, namely the $\mathrm{ACR}^{1}$ phantom in the US and $\mathrm{CDMAM}^{2}$ in the EU. To assess breast imaging technologies such as DBT and contrast-enhanced mammography, there is a need for realistic, 3-D phantoms and new evaluation procedures. To help address this need, this study developed a breast phantom with three key components: a principal section with realistic breast anatomy, anthropomorphic lesions for task-based assessment, and a uniform region for conventional QC purposes. This phantom provided several major advancements over previous generations. This was the first physical rendition of the virtual phantoms from the Duke XCAT Breast Cohort II with higher resolution. In terms of materials, this study achieved targeted $\mathrm{x}$-ray attenuation of fibroglandular tissue using new commercial and custom-doped photopolymer ink. A process of voxelized and dithered photopolymer inkjet printing preserved fine details and subtle tissue gradations. Furthermore, this study introduced several new lesion or task features, including multimaterial anthropomorphic masses, iodine-doped photopolymer printing, calcifications matched to optical scan ground truth, and metal ink sticker QC objects.

There are several broad implications arising from this study. First, these "patient-based" phantoms inherently mimic anatomy because they are based on high-resolution breast CT scans of human subjects. Although this study was based on a single virtual phantom, the process can be applied to other virtual subjects to create phantom "families" to represent anatomical variation. In a separate study, this was demonstrated by assessing lesion detectability in three different phantoms with thickness ranging from 5 to $8 \mathrm{~cm}$ and volumetric breast density from $10 \%$ to $39 \% .{ }^{23}$ Such realism and diversity may play an important role when evaluating the clinical performance of FFDM and DBT systems. Second, the addition of masses and calcifications represent a step toward task-based assessment within a real patient scenario. In a departure from the status quo of subjective scoring, automated QC methods such as model observers may be used to quantify detectability for a relatively small number of lesion tasks and do so in the context of different anatomical backgrounds. Finally, the uniform region was designed for various traditional QC evaluation tasks, including quantitative metrics such as MTF, noise power spectrum, or ASF, as well as qualitative metrics such as resolution line pairs. However, more work is needed to integrate those metrologies to account for the small size of the uniform region and any residual, nonhomogeneous texture caused by the printing process. By acquiring both traditional and new measures of image quality in the same image, one can study their relationships, which should facilitate adoption of these new phantoms and new procedures.

In addition to our work, many other physical breast phantoms have been proposed in recent years. An evaluation of the CIRS BR3D Model 020 phantom, ACR FFDM accreditation phantom, Penn phantom, and Quart mam/digiEPQC phantom found these phantoms to be unacceptable to evaluate DBT systems. ${ }^{6}$ Most of these commercial DBT phantoms are 


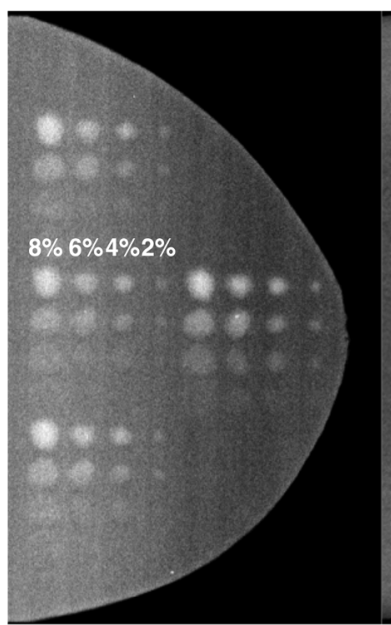

(a)

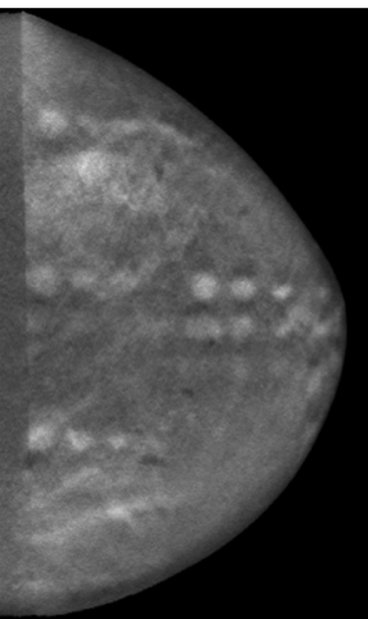

(b)

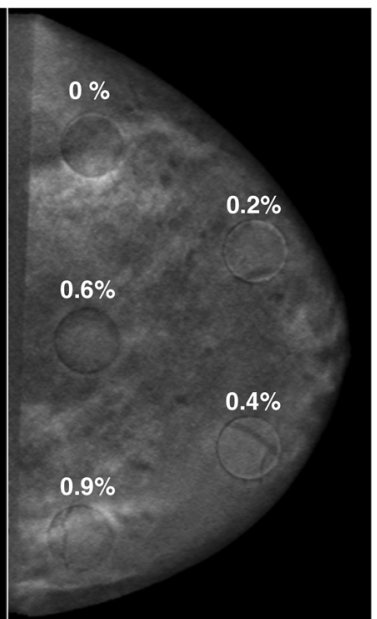

(c)

Fig. 7 lodinated lesion insert: (a) mammography image of the mass insert by itself to accentuate lesion appearances, $39 \mathrm{kVp}$, (b) DBT reconstructed slice image of the mass insert sandwiched in middle depth of the whole breast phantom, $49 \mathrm{kVp}$, and (c) DBT reconstructed slice of iodinated discs sandwiched in whole breast, $49 \mathrm{kVp}$. lodine concentrations are indicated in wt. \%.

fundamentally limited by their uniform background, which cannot fully evaluate 3 -D imaging performance. In addition to the ACR FFDM and quart, other uniform DBT phantoms include the CIRS Model 021, Gammex Modular DBT, Artinis EU DBT Test Set, and Phantom Laboratory Tomophan Phantom. To address the uniform background limitation, several newer phantoms introduced heterogeneous texture, such as the CIRS BR3D, Penn phantom, Leeds Voxmam, and some research phantoms; $;^{5,8,9,24}$ however, they simplify texture as binary, random, and/or procedurally generated patterns that are still readily distinguishable from the real breast. Moreover, only a

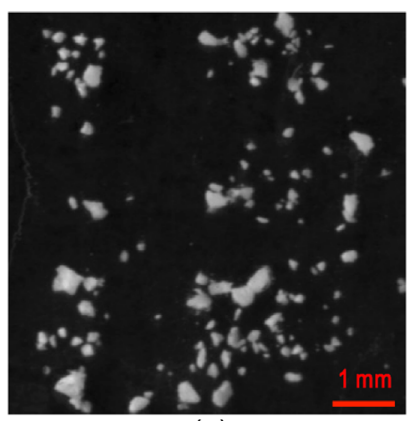

(a)

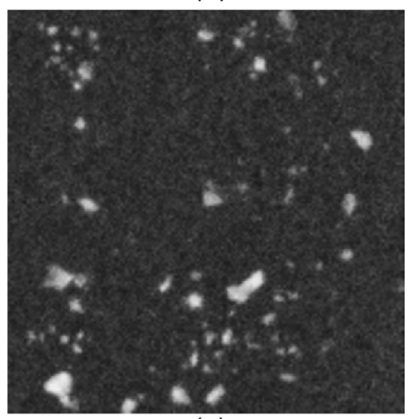

(c)

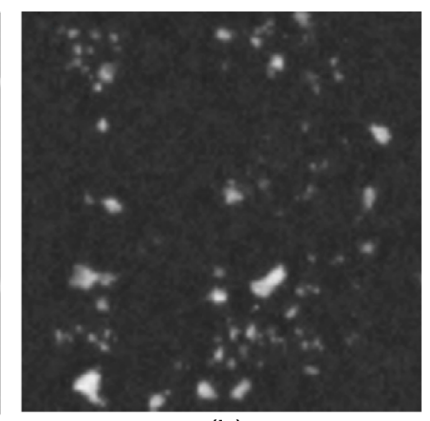

(b)

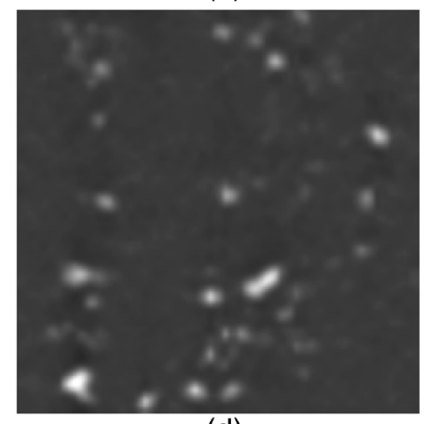

(d)
Fig. 8 Calcification insert images, showing a cropped $6 \times 6 \mathrm{~mm}$ region: (a) optical scan used as ground truth, (b) mammography, (c) magnification view, and (d) DBT reconstructed slice. few research phantoms contain realistic tasks for breast cancer detection, such as Ref. 24 that included calcifications from the Leeds Voxmam and 3-D printed anthropomorphic masses, and Ref. 8 that added hand-crushed calcifications and inkjetprinted masses.

The physical phantoms previously presented by our group also did not include any lesion targets aside from 2-D inserts. ${ }^{8,17}$ We now introduce several types of custom lesions and tasks to allow for nuanced study of how they interplay with varying local volumetric breast density and texture. First, microlobulated masses were arranged in contrast-detail patterns, but the masses are readily customizable including their shape, size, contrast, number, and spatial location, ${ }^{25}$ all while retaining voxel-level ground truth because of the integrated, multimaterial printing process. Second, we used the same process with custom ink to create iodinated lesions, the first ever created using 3-D printing. This is a major advancement over the current commercially available iodinated targets, which are limited to uniform cylinders of resins or just liquid. Third, we reintroduced the use of manually crushed calcifications ${ }^{15}$ but added registration with high-resolution optical scanning to provide ground truth for calcification morphology and location. Finally, metal ink stickers were used to evaluate system resolution as they have a finer resolution and higher contrast than achievable using photopolymer 3-D printing. The metal ink is equivalent to 1 to 2 half value layers of attenuation at mammographic energies, so in addition to line pairs, this process may also be used to create high-contrast objects such as for measuring MTF or ASF.

Another problem of our earlier phantom versions was the limited contrast range of $36 \%$ to $64 \%$ volumetric breast density using the commercial photopolymer inks TangoPlus and VeroWhitePlus. ${ }^{26}$ We raised the upper end to full glandular density with the new commercial ink VeroPureWhite and went beyond with custom, tungsten-doped ink. The doping agents investigated to increase the radiographic density were chosen because they had $k$-edges well outside the mammography energy range $(\mathrm{W}=70$ and $\mathrm{Zn}=10 \mathrm{keV})$, were readily available, and were miscible in the photopolymer ink. For contrastenhanced imaging, iodobutane was chosen for similar reasons. 

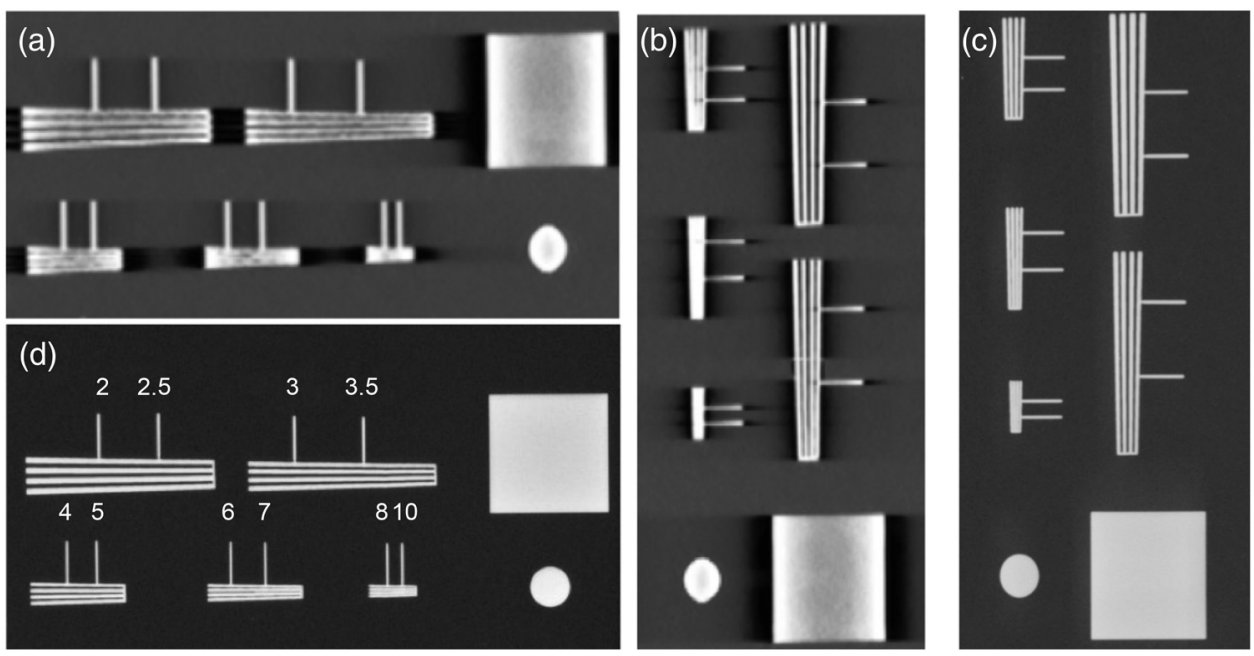

Fig. 9 Metal sticker test objects used to measure standard QC metrics: (a) DBT reconstructed slice image with line pairs oriented parallel to tube motion direction $(4 \mathrm{lp} / \mathrm{mm})$, (b) DBT reconstructed slice with line pairs oriented perpendicular to tube motion direction $(4 \mathrm{lp} / \mathrm{mm})$, (c) magnification view $(6.5 \mathrm{lp} / \mathrm{mm})$, and $(\mathrm{d})$ mammography image $(6.5 \mathrm{lp} / \mathrm{mm})$. Numeric line pair indicators are superimposed on (d) for display purposes only. The square and circle objects were not used in this study.

There are limitations to our current phantom design. Our current inks can achieve or exceed the attenuation of $100 \%$ fibroglandular tissue because the addition of substances is limited only by solubility or miscibility. However, we cannot yet achieve volumetric breast density equivalent below $36 \%$. We have tried to achieve low attenuation by adding the surfactant Span 60 and sonicating to create bubbles, without success. ${ }^{27}$ Our group and others have tried printing only one compartment such as the glandular tissue, then backfilling the adipose portions manually with more tissue equivalent materials such as wax, but this requires greatly simplifying the anatomy and may still result in unacceptable levels of bubbles or artifacts. ${ }^{5,15}$ In an analysis of 3-D printed materials for breast phantoms, acrylonitrile butadiene styrene used in fused deposition modeling printing was shown to maintain an absorption close to that of adipose tissue throughout the mammographic energy range ${ }^{28}$ however, there remains considerable work to develop adipose equivalent materials that can comply with the inkjet and curing process. For simplicity in this study, phantom materials were evaluated with a typical radiographic technique $(\mathrm{W} / \mathrm{Rh}$ at $28 \mathrm{kVp})$. However, previous work validated that these photopolymers maintained their relative attenuation compared to tissue-equivalent reference materials over the full mammographic energy range. ${ }^{15}$ In addition, the phantoms in this study focus on a single-subject anatomy. In other studies, however, three other models have been fabricated based on subjects with different breast size, thickness, and density. ${ }^{23}$ Finally, although various lesion tasks are presented, they may need to be refined for clinical application. Currently, lesions are restricted to the middle depth, but the phantom can be made in thinner cross sections such that lesions may be sandwiched at other depths. Similarly, calcifications are confined to a 2-D plane just like conventional phantoms, but potentially multiple layers can be stacked with spacer slabs to depict a 3-D cluster. Finally, protocols would need to be developed for task-based assessment in anthropomorphic physical phantoms, but that is beyond the scope of this study. The contrast-enhanced lesions have yet to be validated on a clinical dual-energy system to accurately create a subtraction image. Future work may be directed toward refining those lesion tasks, such as to create spiculated masses ${ }^{25}$ or to create fixed calcification patterns using 2-D inkjet printing ${ }^{17}$ to integrate calcifications into the breast anatomy.

\section{Conclusion}

This study represents significant improvements to our current phantom design. The commercial product VeroPureWhite and the custom, tungsten-doped Jf Flexible resin were presented to represent fibroglandular tissue. The commercial TangoPlus and the third party Jf Flexible resin without dopant were used to represent low-density adipose tissue. The modular approach allows for maximum versatility of the phantom including healthy tissue, lesions, contrast-enhanced lesions, and calcifications within the anthropomorphic region as well as a space for standard QC metric evaluation in the uniform chest wall region. Our fundamental goal is to create techniques that can provide flexible, customizable, and affordable options to fabricate phantoms that suit the needs of different researchers.

\section{Disclosures}

Dr. Lo has a license agreement with Gammex Inc./Sun Nuclear regarding intellectual property that may be related to this study. He has a conflict of interest management plan that has been approved by the Duke University School of Medicine Research Integrity Office.

\section{Acknowledgments}

We would like to thank Steve Glick for providing breast CT data used for the virtual breast model and for many helpful discussions, Anne Li for analysis of metal ink stickers, Charles Cowart and Greg Sturgeon for design and fabrication of phantoms, and Thomas Sauer who provided the virtual mass model. As this paper is the culmination of a long-term project, incremental results were previously presented at several SPIE proceedings. ${ }^{27,29,30}$ This research was funded in part by NIH/ NIBIB R21EB025549; the Charles E. Putman M.D. Seed Fund of the Department of Radiology, Duke University School of 
Medicine; and the Pratt Engineering Undergraduate Research Fellows Program, Pratt School of Engineering, Duke University.

\section{References}

1. R. McLelland et al., "The American College of Radiology Mammography Accreditation Program," Am. J. Roentgenol. 157, 473-479 (1991).

2. K. Bijkerk, J. Lindeijer, and M. Thijssen, Manual, CDMAM-Phantom, Department of Diagnostic Radiology, University Hospital, Nijmegen (1995).

3. US FDA, "MQSA national statistics," 2017, https://www.fda.gov/ radiation-emittingproducts/mammographyqualitystandardsactand program/facilityscorecard/ucm113858.html (accessed 23 May 2017).

4. R. E. van Engen et al., "Digital mammography update. European protocol for the quality control of the physical and technical aspects of mammography screening," in S1, Part 1: Acceptance and Constancy Testing, in European Guidelines for Quality Assurance in Breast Cancer Screening and Diagnosis, 4th ed., Supplements, B. M. Perry et al., Eds., European Commission, Office for Official Publications of the European Union, Luxembourg, pp. 1-54 (2013).

5. A. K. Carton et al., "Development of a physical 3D anthropomorphic breast phantom," Med. Phys. 38(2), 891-896 (2011).

6. C. Brunner et al., "Evaluation of various mammography phantoms for image quality assessment in digital breast tomosynthesis," in Breast Imaging, A. A. Maidment, P. Bakic, and S. Gavenonis, Eds., Springer, Berlin, Heidelberg, pp. 284-291 (2012).

7. L. Cockmartin, H. Bosmans, and N.W. Marshall, "Comparative power law analysis of structured breast phantom and patient images in digital mammography and breast tomosynthesis," Med. Phys. 40(8), 081920 (2013).

8. L. C. Ikejimba et al., "A novel physical anthropomorphic breast phantom for 2D and 3D x-ray imaging," Med. Phys. 44(2), 407-416 (2017).

9. J. G. Mainprize et al., "Development of a physical 3D anthropomorphic breast texture model using selective laser sintering rapid prototype printing," Proc. SPIE 10573, 105730A (2018).

10. S. Vecchio et al., "A novel approach to digital breast tomosynthesis for simultaneous acquisition of 2D and 3D images," Eur. Radiol. 21(6), 1207-1213 (2011).

11. V. Filippou and C. Tsoumpas, "Recent advances on the development of phantoms using 3D printing for imaging with CT, MRI, PET, SPECT, and ultrasound," Med. Phys. 45(9), e740-e760 (2018).

12. D. W. Erickson et al., "Population of 224 realistic human subject-based computational breast phantoms," Med. Phys. 43(1), 23-32 (2016).

13. C. M. Li et al., "Methodology for generating a 3D computerized breast phantom from empirical data," Med. Phys. 36(7), 3122-3131 (2009).

14. J. Rajagopal et al., "Evaluation of statistical breast phantoms with higher resolution," Proc. SPIE 10573, 1057307 (2018).

15. N. Kiarashi et al., "Development of realistic physical breast phantoms matched to virtual breast phantoms based on human subject data," Med. Phys. 42(7), 4116-4126 (2015).

16. L. Ikejimba et al., "A quantitative metrology for performance characterization of five breast tomosynthesis systems based on an anthropomorphic phantom," Med. Phys. 43(4), 1627-1638 (2016).

17. L. C. Ikejimba et al., "Assessing task performance in FFDM, DBT, and synthetic mammography using uniform and anthropomorphic physical phantoms," Med. Phys. 43(10), 5593-5602 (2016).

18. J. Solomon et al., "Comparison of low-contrast detectability between two CT reconstruction algorithms using voxel-based 3D printed textured phantoms," Med. Phys. 43(12), 6497-6506 (2016).
19. W.-R. Ng, "Fast and inexpensive fabrication of terahertz components via adaptation of rapid prototyping technology," MS Thesis, Department of Electrical and Computer Engineering, University of Arizona, p. 89 (2012).

20. P. C. Johns and M. J. Yaffe, "X-ray characterisation of normal and neoplastic breast tissues," Phys. Med. Biol. 32(6), 675-695 (1987).

21. N. Kiarashi et al., "Impact of breast structure on lesion detection in breast tomosynthesis, a simulation study," J. Med. Imaging 3(3), 035504 (2016).

22. A. J. Evans, Breast Calcification: A Diagnostic Manual, 1 ed., Greenwich Medical Media, San Francisco; London (2002).

23. C. Cowart, "Task-based assessment of digital breast tomosynthesis: effect of anatomy from multiple anthropomorphic 3D printed phantoms," Master's thesis, Duke University (2017).

24. L. Cockmartin et al., "Design and application of a structured phantom for detection performance comparison between breast tomosynthesis and digital mammography," Phys. Med. Biol. 62(3), 758-780 (2017).

25. T. J. Sauer et al., "Detectability of artificial lesions in anthropomorphic virtual breast phantoms of variable glandular fraction," Proc. SPIE 10132, 101321X (2017).

26. A. Nolte et al., "A second generation of physical anthropomorphic 3D breast phantoms based on human subject data," Proc. SPIE 9033, 90331Y (2014).

27. C. Zhao et al., "Third generation anthropomorphic physical phantom for mammography and DBT: Incorporating voxelized 3D printing and uniform chest wall QC region," Proc. SPIE 10132, 101321Y (2017).

28. D. Ivanov et al., "Suitability of low density materials for 3D printing of physical breast phantoms," Phys. Med. Biol. 63(17), 175020 (2018).

29. A. Rossman et al., "3D printed anthropomorphic physical phantom for mammography and DBT with high contrast custom materials, lesions, and uniform chest wall region," Proc. SPIE 10573, 105730C (2018).

30. D. Sikaria et al., "Second generation anthropomorphic physical phantom for mammography and DBT: incorporating voxelized 3D printing and inkjet printing of iodinated lesion inserts," Proc. SPIE 9783, 978360 (2016).

Andrea H. Rossman received her BSE degree in biomedical engineering from Pratt School of Engineering, Duke University. Currently, she is a research specialist in the Morphomic Analysis Group of the University of Michigan. She will begin as a first year medical student in the summer of 2019.

Michael E. Gehm is an associate professor of electrical and computer engineering. His research is in computational and compressive sensing and measurement, with rapid-prototyping as a means of creating advanced electromagnetic structures.

Ehsan Samei is a professor of radiology, medical physics, biomedical engineering, physics, and electrical and computer engineering at Duke University. His research includes x-ray imaging, theoretical imaging models, simulation methods, and experimental techniques in medical image formation, analysis, assessment, and perception.

Benjamin J. Wiley is an associate professor of chemistry at Duke University. His lab focuses on making new nanomaterials by controlling the assembly of atoms in solution, and exploring applications for nanomaterials in medicine, catalysis, plasmonics, and electronics.

Joseph Y. Lo is a professor of radiology, biomedical engineering, electrical and computer engineering, and medical physics at Duke University. He is a director of the Carl E. Ravin Advanced Imaging Laboratories (RAI Labs). His group investigates machine learning for breast and CT imaging, and develops virtual and physical phantoms to evaluate imaging performance in virtual clinical trials. 